\title{
CIENCIA, TÉCNICA Y DICCIONARIO EN LA PRIMERA MITAD DEL SIGLO XIX: INCUBANDO REVOLUCIONES*
}

\author{
SCIENCE, TECHNIQUE AND DICTIONARY IN THE FIRST HALF OF THE \\ 19TH CENTURY: INCUBATING REVOLUTIONS
}

\author{
Cecilio Garriga Escribano \\ Universitat Autònoma de Barcelona \\ Grupo Neolcyt \\ Cecilio.Garriga@uab.cat
}

Recibido: $13 / 12 / 2018$

Aceptado: 10/03/2019

\begin{abstract}
Resumen
Lengua y ciencia son dos realidades de un mismo fenómeno, el avance del conocimiento. Los diccionarios son testimonios de los cambios que se producen en la lengua de la ciencia, pero en el caso del español moderno influyen factores diferenciales. En este trabajo se analiza la posición de la Academia ante el léxico científico y técnico a partir de lo manifestado en los prólogos de las ediciones de la primera mitad del siglo XIX, se comprueba esa posición con los datos disponibles acerca de los vocabularios científicos y técnicos en esas ediciones, y se contrasta con la situación de la ciencia española en ese período a partir de los análisis de la historia de la ciencia. Finalmente, se aplican los esquemas de la his-
\end{abstract}

\begin{abstract}
Language and science are two realities of the same phenomenon, the progress of knowledge. Dictionaries are testimonies of the changes that take place in the language of science, but in the case of modern Spanish differential factors influence. In this work, the position of the Real Academia before the scientific and technical lexicon is analyzed from what is manifested in the prologues of the first half of the 19th century editions, it is verified with the available data about the scientific and technical vocabularies in those editions, and it is contrasted with the situation of Spanish science in that period through the analysis of the history of science. Finally, the schemes of the history of science
\end{abstract}

\footnotetext{
* Este estudio se enmarca en el proyecto de investigación El lenguaje especializado del español contemporáneo: 1884-1936, financiado por la Agencia Estatal de Investigación (PGC2018-093527-B-I00) y desarrollado por Neolcyt, integrado en el Grupo "Lexicografía y Diacronía" reconocido como grupo consolidado por la Generalitat de Catalunya (2017SGR-1251), y que forma parte de la Red Temática "Lengua y ciencia" (FFI2015-68705-REDT).
}

Para citar este artículo / To cite this article: Garriga, Cecilio (2019). Ciencia, técnica y diccionario en la primera mitad del siglo XIX: incubando revoluciones.Azorín, Dolores; Clavería, Gloria y Jiménez Ríos, Enrique (Eds.): ELUA: El diccionario de la Academia y su tiempo: lexicografí, lengua y sociedad en la primera mitad del siglo XIX, Anexo V, págs. 47-63.

Enlace/Link: http://dx.doi.org/10.14198/ELUA2019.ANEXO5.02 
toria de la ciencia para ensayar una explicación de la situación de la lengua de la ciencia y de la técnica en el diccionario en esa etapa.

PALABRAS CLAVE: Lengua de la ciencia, terminología, diccionario, Real Academia Española, siglo XIX are applied to essay an explanation of the situation of the language of science and technique in the dictionary at that stage

KEY WORDS: Language of science, terminology, dictionary, Real Academia Española, 19 th century

\section{INTRODUCCIÓN}

Se suele admitir que el diccionario es el notario de la lengua, y que la lengua es a su vez reflejo de la sociedad a la que sirve de instrumento. Esta afirmación se acentúa si se piensa en la parte de la lengua que sirve de expresión a la ciencia y a la técnica, un ámbito especialmente dinámico a partir del siglo XVIII, con el avance del conocimiento, las aplicaciones técnicas a la industria y el desarrollo científico. Esta actividad genera nuevas necesidades léxicas porque hay que nombrar nuevos conceptos, substancias, inventos, aparatos, etc.

Aunque España no genera esos conocimientos en la mayor parte de los casos, sí que se divulgan a través de las traducciones, de los manuales, de las disposiciones legales, y fuerzan al español a crear o adaptar las palabras para expresarlos, en muchas ocasiones con la inminencia que el conocimiento científico y técnico requiere. El diccionario, en estos casos, aún suele retrasar más la incorporación de estas palabras, porque se consideran demasiado especializadas, o neológicas, o extranjeras...

Para estudiar la historia del español en esta época, y de su lexicografía, hay que atender, por tanto, a diversas variables: las circunstancias por las que pasa el diccionario en la primera mitad del siglo XIX, el desarrollo de las ciencias y técnicas que irrumpen en esa época con una fuerte penetración en la sociedad, y por tanto en la lengua, y todo ello en el marco de la situación histórica que condiciona tanto la ciencia como el diccionario.

Los historiadores de la ciencia han descrito bastante bien el siglo XIX, y la hipótesis aquí es que se pueden aplicar las mismas etapas al desarrollo del diccionario que al avance de las ciencias. Para ello, se comprobará, por una parte, el pensamiento lingüístico que se expresa en los prólogos de las diferentes ediciones, y por otra se examinará una serie de vocabularios que ya han sido estudiados en trabajos previos. Finalmente se vuelve a la historia de la ciencia para comparar las etapas descritas con las establecidas por los estudios que tienen como base la lengua de la ciencia.

La evidencia es que las ediciones de esta primera mitad del siglo XIX taponan la entrada de las voces de ciencias y artes, y que este aspecto se convierte en uno de los más criticados, como reflejo del atraso del diccionario. Así aparece una serie de diccionarios al margen de la Academia hacia el final de ese período que utilizan este aspecto de las lenguas de las ciencias con el afán acumulativo que caracteriza a la lexicografía enciclopédica, convirtiéndose en un reclamo y a la vez presionando a la Corporación que empieza a atender esta reclamación a partir de la $11^{\mathrm{a}}$ edición (RAE 1869), y a modernizar su léxico en las ediciones de la segunda mitad del siglo.

Así que la presencia o ausencia de las voces técnicas en el diccionario obedece, por un lado, al desarrollo de las ciencias y de las técnicas, y por otro a la vida interna de la Academia. Y para entender todo ello, se hace necesario volver la vista al pasado inmediato, y arrancar del estado de la lengua de la ciencia en el siglo anterior. 


\section{LA LENGUA DE LA CIENCIA Y EL DICCIONARIO EN EL SIGLO XVIII}

La idea que existe acerca del lenguaje científico y técnico en relación al español debe mucho a los estudios de Lázaro Carreter sobre el léxico en el siglo ilustrado. En su clásico ensayo Las ideas lingüísticas en España durante el siglo XVIII, en un capítulo titulado "Neologismo y purismo", sentenciaba que "la falta de léxico es un mal que arrastra nuestro idioma desde el Renacimiento hasta nuestros días" (Lázaro Carreter 1949 [1985]: 284). La afirmación se basaba en la escasa actividad científica en España, frente a los magníficos frutos que había dado la creación literaria: "Por la época en que Galileo, Copérnico y Kepler dotaban al mundo de leyes naturales y matemáticas, España producía sus más asombrosas obras literarias, quedando al margen de aquellas inquietudes".

Sin embargo, los avances de la historia de la ciencia y de la historia de la lengua de la ciencia permiten matizar esta idea. Hoy sabemos que sí hubo un desarrollo científico importante desde el Renacimiento, como no podía ser de otra manera, en un país que era una potencia marítima y militar. Ciertamente en unos campos más que en otros, pero los trabajos coordinados por López Piñero (2002) para los siglos XVI y XVII, y por Peset (2002) para el XVIII dan una imagen más real y objetiva de la situación y los alcances en los diversos campos de la ciencia y la técnica. Por su lado, los trabajos realizados por el equipo del DICTER muestran que había textos importantes en el arte militar, la astronomía, la construcción, la cosmografía, la destilación, la fortificación, la legislación, la maquinaria, las matemáticas, la metalurgia, la náutica o la óptica. En cualquier caso, ese desarrollo científico y técnico dio lugar a la publicación de tratados y ensayos, en los que la preocupación por la lengua estaba ya presente (Mancho 2008: 315). Así que se fue formando un vocabulario especializado que en muchos casos se tradujo en la elaboración y publicación de diccionarios técnicos, algunos de ellos de gran repercusión (García-Macho 2014).

Esta realidad se percibe ya en el primer diccionario académico (RAE 1726-1739), que tiene que determinar qué parte de ese vocabulario acoge en sus páginas. Y la Academia decidió que solo iba a incluir las voces que fueran de uso común, y que cuando acabara ese primer diccionario, compondría uno de voces de ciencias y artes, que quedó solo en proyecto. Aun así, los estudios realizados acerca del diccionario de Autoridades muestran que el vocabulario especializado que se encuentra en sus páginas es considerable, y que se utilizaron importantes obras especializadas, tratados y diccionarios, como fuente del trabajo académico (Freixas 2010: 370). Esa tendencia se mantuvo en el único tomo de la segunda edición de Autoridades, en la que aumenta la presencia de voces de ciencias y artes (Pascual Fernández 2013).

Más explícito fue Esteban Terreros (1786), en su Diccionario de la lengua castellana con voces de ciencias y artes, quien ya recogía en el propio título la intención de reunir este tipo de voces e incluirlas en su diccionario. Aunque Lázaro Carreter (1949 [1985]: 286) opinaba que el diccionario de Terreros era "un panteón, un archivo de formas, que solo de manera muy limitada podía intervenir para remediar la falta de léxico técnico español”, y que "no podía satisfacer la urgente y rapidísima tarea de verter obras y obras en un tiempo brevísimo, de traducir voces correspondientes a conceptos nuevos", lo cierto es que, a juzgar por el recuento de Alvar Ezquerra (1987: XIII), era una buena representación del léxico especializado existente en el español y supuso un avance sobre el vocabulario técnico recogido en Autoridades (Azorín y Santamaría 2004; Raab 2018: 534). 
Esta era la situación cuando en la segunda mitad del siglo XVIII España pugna por retomar el paso de la ciencia europea. El impulso dado por los monarcas de la Casa de Borbón, y en especial por Carlos III, a las ciencias, hace que se traduzcan los textos más importantes de cada disciplina, y que se escriban manuales para la enseñanza de las ciencias. Esta actividad conlleva la necesidad de crear nuevo vocabulario en cada una de las disciplinas, ya sea mediante los procedimientos morfológicos propios del español o a través de la adopción de préstamos, fundamentalmente del francés. La sensibilidad hacia la lengua era grande, como demuestra la Oración sobre la necesidad de unir el estudio de la literatura a las ciencias, en la que Jovellanos expresaba la necesidad de que la ciencia, para ser útil, debía transmitir su saber al ciudadano en un idioma comprensible (Galera 2002: 62).

Los autores y traductores de tratados y manuales se ven en la circunstancia de superar los límites del léxico oficial, tal como lo expresan en muchos pasajes de sus textos. Así, Pedro Gutiérrez Bueno, traductor de la Nueva nomenclatura química de Morveau, Lavoisier, Berthollet y Fourcroy (1788: II-III), se disculpaba por utilizar palabras que no estuvieran autorizadas "por los mejores diccionarios de nuestra lengua"; Melchor de Guardia y Ardévol, traductor de los Elementos de química teórica y práctica (Morveau, Maret y Durande 1788: s. p.), hablaba de la dificultad de esta tarea:

Qualquiera que se dedique á traducir conocerá fácilmente la dificultad de traducir bien los libros de una ciencia en que apénas se halla ninguno escrito en nuestra lengua y que acaba de padecer una revolucion en el lenguage, lo que parece que me autorizaria alguna vez para introducir el nombre de alguna cosa que no le tenga entre nosotros.

Y este problema se planteaba no solo en relación al vocabulario de la química. Cristóbal Cladera, traductor del Diccionario universal de física de Brisson (1796: XXVII), hablaba de la dificultad para traducir nuevos conceptos que "por su novedad, ó porque no se han cultivado en España, no tienen su correspondencia exâcta en nuestra lengua" (Garriga 1998: 183), y Benito Bails (1802: prólogo), en su Diccionario de arquitectura civil, avisaba de que: "el que reparare algún artículo que no concuerde con el Diccionario de la Real Academia Española, tenga presente que no definimos voces de la lengua común, sino testimonios de un arte".

Así pues, mientras el español tenía que ensanchar sus límites para satisfacer las necesidades de científicos, profesores y traductores, el diccionario constreñía las posibilidades expresivas de la lengua de la ciencia. Lázaro Carreter (1949 [1985]: 287) explica cómo Capmany emprendía el esfuerzo de renovar el léxico científico del español a través de su Diccionario francés-español (1801). Mientras tanto, la Academia se debatía por avanzar en la segunda edición del Diccionario de Autoridades, a la vez que iba dando a la luz nuevas ediciones del Diccionario de la lengua castellana reducido a un tomo para su más fácil uso (Álvarez de Miranda 2001: 48).

\section{LA LENGUA DE LA CIENCIA Y EL DICCIONARIO EN LA PRIMERA MITAD DEL SIGLO XIX}

Para estudiar la relación entre la lengua de la ciencia y el diccionario es necesario atender al desarrollo de ambos aspectos. Por ello prestaré atención en primer lugar a lo que el propio diccionario dice de las voces de la ciencia en los prólogos de sus ediciones, 
y en segundo lugar examinaré la presencia de esas voces en el cuerpo del diccionario, a la luz de los estudios que sobre determinados vocabularios se han realizado.

\subsection{La lengua de la ciencia en los prólogos académicos}

Aunque uno de los primeros estudios panorámicos que se realizaron acerca del diccionario académico tomó como base los prólogos de las ediciones (Alvar Ezquerra 2002), conviene aquí detenerse en este aspecto ya que, como es sabido, es en los prólogos de los diccionarios donde se pueden encontrar explícitas algunas de las decisiones técnicas que toman los lexicógrafos (San Vicente y Lombardini 2012: 482). También es cierto que a veces determinados comentarios que aparecen en los prólogos despiertan unas expectativas que no se corresponden con lo que ocurre en el interior del diccionario. En cualquier caso, este es un aspecto que conviene valorar siempre.

El diccionario abre el siglo XIX con la publicación de la $4^{\text {a }}$ edición (RAE 1803). En su parte inicial no se halla una alusión explícita a las voces de ciencia y artes, aunque se habla de la incorporación de voces en todas las letras del diccionario. Como ha señalado Álvarez de Miranda (2000: 49), en esta edición deja de considerarse el diccionario en un tomo como una solución provisional mientras no se dispusiera de la $2^{\mathrm{a}}$ edición de Autoridades, porque la Academia había abandonado ya la idea de publicarla.

En la $5^{\mathrm{a}}$ edición (RAE 1817) aparece la primera referencia a las voces especializadas, pero no en cuanto a las nuevas palabras que se incorporan, sino a su tratamiento. Se habla de la simplificación de las definiciones "especialmente en los artículos que pertenecen a ciencias naturales". Y de nuevo la $6^{\mathrm{a}}$ (RAE 1822) omite cualquier referencia al vocabulario especializado, aunque hace alusión en varias ocasiones al aumento de voces del diccionario. Sin embargo, lo cierto es que la nomenclatura académica reduce el número de voces en 2.032, pasando de 58.886 a 56.854, según los datos que se extraen del Nuevo tesoro lexicográfico ${ }^{1}$.

La $7^{\mathrm{a}}$ edición académica (RAE 1832) sí que expresa explícitamente la preocupación por las voces especializadas, pero no por el aumento, sino de nuevo por la corrección de las definiciones con criterios lexicográficos, igual que se manifestaba en el prólogo de la $5^{\text {a }}$ edición. Es interesante seguir la argumentación sobre el nivel de especialización con que deben ser redactadas las definiciones:

(...) han llamado su atención las voces tomadas de artes y ciencias, y en particular de los diversos ramos que comprenden las ciencias naturales. Las definiciones de estas en las primeras ediciones no pudieron menos de resentirse de la imperfeccion de las mismas ciencias en aquel tiempo, y despues al corregirlas se convirtieron no pocas de ellas en explicaciones ó descripciones demasiado largas, porque se consideró que todavía no eran muy conocidos los objetos de que se trataba. Pero como por una parte se ha extendido ya mas la afición á este género de estudios, y por otra solo á los tratados científicos corresponden semejantes explicaciones, ha intentado la Academia acortarlas, poniéndolas al mismo tiempo con la posible exactitud. La empresa a la verdad es difícil, porque si es cierto que el Diccionario no debe presentarlas como las de un profesor á sus discípulos, tambien lo es que no teniendo por lo común idea perfecta de semejantes cosas sino los que

1 Agradezco a Rodríguez Marín que me proporcionara los datos de Octavio Pinillos a partir de la informatización del NTLLE. Si no se dice lo contrario, todos los datos sobre el número de entradas de cada edición proceden de esta fuente. 
se han dedicado á observarlas profundizando en la materia, se tropieza con el escollo, ó de que las definiciones sean vagas, inexactas y acaso falsas, cuando se acomodan á la idea vulgar, ó de que separándolas de ella, sean oscuras para un gran número de lectores. La Academia en obsequio de los mismos ha sacrificado á veces lo segundo á lo primero; más en general ha procurado no faltar á la exactitud cuidando solo de evitar en las definiciones los términos meramente científicos, que por lo mismo no constan en el Diccionario. Así entiende que ayuda á los profesores á conocer mejor el nombre castellano de lo que acaso no ha llegado á su noticia sino por obras extrangeras, y á los demás lectores á formar la idea verdadera y precisa de lo que conocen imperfectamente.

La Academia sigue manteniendo que no está dispuesta a admitir las voces que no estén en los autores clásicos, o que no tengan un uso reconocido, así como "los nombres caprichosos y pasageros de trajes y modas", y avisa de que "ha excluido infinitas voces técnicas de ciencias, artes y oficios que no pertenecen al lenguaje comun, único objeto el Diccionario". El resultado es una reducción de 6.187 entradas en la nomenclatura de esta $7^{\mathrm{a}}$ edición ${ }^{2}$.

La $8^{a}$ edición (RAE 1837) mantiene el argumento, ahora referido especialmente a las voces de ciencia y de técnica formadas por raíces clásicas:

Habiéndose aumentado desmedidamente la nomenclatura de origen griego, aplicada no solo á varias ciencias, sino á los diversos ramos que comprende cada una, acuden los curiosos al Diccionario en busca de los nombres de aquella procedencia, y no hallándolos en él, lo acusan de pobre y diminuto. La Academia se ve por tanto en la precision de advertir, que tales nombres pertenecen ménos al caudal de los idiomas vulgares, que al lenguaje técnico peculiar de las ciencias á que se refieren. Por lo mismo no se juzga autorizada para darles lugar en su Diccionario, hasta tanto que el transcurso del tiempo los va haciendo familiares, y el uso comun los adopta y prohija. Así se ha conducido en cada una de sus varias ediciones, según han ido adquiriendo cierta especie de naturalización, é incorporándose, por decirlo así, en el fondo general de la lengua.

Este argumento recuerda poderosamente el utilizado por Capmany (1817: XXII) en el prólogo de su Nuevo diccionario francés español cuando justificaba la correcta adaptación de los préstamos del francés: "son voces de la lengua docta, aunque no lo sean del diccionario. La necesidad las autoriza y la analogía las prohija, y españoliza”.

Pero la $9^{a}$ edición (RAE 1843) es la que presenta una mayor atención en el prólogo a las voces de la ciencia y de la técnica. Ciertamente las críticas a la Academia por la parquedad de su vocabulario arreciaban, y en especial en lo que a las voces de ciencias y artes se refería (Seco 1987). Así que la Corporación se siente en la necesidad de explicar, como ya hacía en la $5^{\text {a }}$ edición (RAE 1817), que estas voces no deben formar parte de los diccionarios generales sino de las obras especializadas:

[...] hay tambien una inmensa nomenclatura de las ciencias, artes y profesiones, cuyo significado deben buscar los curiosos en los vocabularios particulares de las mismas: tales voces pertenecen á todos los idiomas y á ninguno de ellos, y si hubieran de formar parte del Diccionario de la lengua comun, (...) sería una obra voluminosa en demasías, semienciclopédica y de difícil adquisición y manejo.

2 Carriet (2017: 41) cifra en 313 los artículos nuevos, 30 de los cuales pertenecen al suplemento. 
Solo cuando estas palabras rebasan el campo al que pertenecen pueden incluirse en el diccionario, porque se consideran parte de la lengua general:

Otros echan de menos en el Diccionario de la lengua castellana la multitud de términos facultativos pertenecientes á las artes y las ciencias, de las cuales solo se debe admitir aquellos, que saliendo de la esfera especial á que pertenecen, han llegado á vulgarizarse, y se emplean sin afectación en conversaciones y escritos sobre diferente materia.

La Academia admite que cada vez es mayor la penetración de frases y voces técnicas en el idioma general, y por eso aumenta su número respecto a la edición anterior. Pero mantiene el criterio de que antes de incluir una voz nueva en el diccionario es necesario comprobar "si la adopción es constante y sostenida, ò solo temporal y transitoria", para no cargar el diccionario de voces efímeras.

El período que aquí se estudia acaba con la $10^{\mathrm{a}}$ edición académica (RAE 1852), en la que se declara que se han introducido nuevos términos procedentes de diferentes ámbitos (administración pública, artes, comercio e industria). A pesar de ello, la Academia admite que pueden faltar algunas "voces novísimas" que ha excluido "bien por demasiado técnicas, bien porque, apenas empleadas por algunos escritores, han caido en completo desuso, ó se han medio naturalizado en Castilla cuando ya se había impreso el pliego donde habrian de colocarse".

Hay que tener en cuenta que entre ambas ediciones, la $9^{\mathrm{a}}$ y la $10^{\mathrm{a}}$, se produce la eclosión de lo que se ha dado en llamar la lexicografía "no académica" (Seco 1987), con la publicación del Nuevo diccionario de la lengua castellana de Salvá (1846) y el Diccionario nacional de Domínguez (1846-47), ambos caracterizados por criticar duramente el vocabulario de la Academia por no incluir las voces que reflejaban los avances de la ciencia y de la técnica ${ }^{3}$.

Así pues, a lo largo de los prólogos de las ediciones de la primera mitad del siglo XIX se observa un cambio en relación a las voces de la ciencia y de la técnica. En la primera etapa el foco está en las definiciones, para encontrar el tono adecuado a un diccionario general, lo que queda explicitado en los prólogos de la $5^{\mathrm{a}}$ (RAE 1817) y la $7^{\mathrm{a}}$ (RAE 1832) ediciones. De hecho, las Reglas para la corrección y aumento del diccionario (RAE 1743) y (RAE 1757) ya mostraban la necesidad de corregir las definiciones de Autoridades especialmente en las voces facultativas (Rodríguez Ortiz y Garriga 2010: 42).

En la segunda etapa, como queda patente en los prólogos de las ediciones $8^{\mathrm{a}}$ (RAE 1837), $9^{\text {a }}$ (RAE 1843) y 10 (RAE 1852), el objetivo es explicar el criterio de inclusión de las voces de ciencias y artes en el diccionario, para justificarse ante la crítica por la escasez de este vocabulario en sus páginas. La idea es que solo deben entrar aquellas voces que se naturalizan y se asientan en la lengua, perdiendo su carácter especializado. Aunque este principio ya se incluyó en las Reglas de 1770 ("de las voces de artes y ciencias solo se han de poner aquellas que estan recibidas en el uso comun de la lengua") (Rodríguez Ortiz y Garriga 2010: 41), vuelve a cobrar actualidad en estas últimas ediciones de la primera mitad del siglo XIX.

3 Sobre el diccionario de Salvá, véase Azorín (2003a) y (2003b), y Álvarez de Miranda (2011); y sobre el de Domínguez pueden verse los estudios de Esparza (1999), Garriga e Iglesia Martín (2010), Quilis Merín (2007), Iglesia (2008), (2011) y (2012), donde se hace referencia especialmente a estos aspectos. 


\subsection{La lengua de la ciencia en el cuerpo del diccionario}

Clavería (2016: 53) clasifica las ediciones académicas del siglo XIX en tres períodos: 1803 y 1817,1822 a 1852, y 1884 a 1899 . Como se puede observar, los dos primeros se ubican prácticamente en la primera mitad del siglo, que es la etapa que aquí se estudia.

Por lo que respecta al primer período, las ediciones $4^{\mathrm{a}}$ y $5^{\mathrm{a}}$ obedecen a criterios claramente diferenciados. La $4^{\mathrm{a}}$ (RAE 1803) tiene como objetivo el aumento de las voces (se registra un crecimiento de 9.399 respecto a la $3^{\mathrm{a}}$ edición (RAE 1791)). Se beneficia para ello de los trabajos de revisión del Diccionario de Autoridades, que alcanzaba hasta la letra $L$. Como se puede esperar, gran parte de este aumento se realiza a expensas de las voces de ciencia y de técnica. Clavería (2016: 63) señala como campos tradicionales el léxico de la náutica, el forense, el militar, pero también los campos más propiamente científicos y técnicos, como la medicina, la arquitectura, la matemática, la química y la física, o la mineralogía. Los oficios están también presentes, como la carpintería, la tintorería, la imprenta, el comercio, etc.

En efecto, en algunos de los estudios realizados por el grupo Neolcyt se observan innovaciones significativas en el léxico técnico. Es el caso de la electricidad, un ámbito que tanta repercusión tendría en el desarrollo científico, industrial y social del siglo XIX, ya que en esta $4^{\mathrm{a}}$ edición se incorporan, por ejemplo, electricidad, eléctrico y electrizar (Moreno Villanueva 2012: 412). Y también lo es el de la química, con la incorporación de oxígeno y gas en el suplemento (Garriga 2003: 105).

La $5^{\mathrm{a}}$ edición (RAE 1817), en cambio, registra un aumento neto de solo 95 voces respecto a la edición anterior. No obstante, las incorporaciones ascenderían a 1.292, según el recuento de Clavería (2018: 40), siendo importante la tarea de revisión realizada, que conlleva la supresión de numerosas entradas, entre las que destacan las marcadas diacrónicamente (Jiménez Ríos 2018: 415).

Afortunadamente los estudios coordinados por Clavería y Freixas (2018) permiten conocer con mucho detalle esta $5^{\mathrm{a}}$ edición académica, y valorarla por las importantes mejoras que experimentó el diccionario. En lo que a las voces especializadas se refiere, una parte importante del esfuerzo se destinó a actualizar definiciones obsoletas, aunque también se producen incorporaciones en áreas como la botánica, la medicina y cirugía, la mineralogía, la zoología, etc. (Gómez de Enterría 2018: 310). Algo parecido ocurre con las voces de la química, en las que no es tan importante el aumento (apenas cinco incorporaciones: ázoe, calórico, concentración, hidrógeno y oxigenar), como la revisión y enmienda de las definiciones de términos tradicionales (agua, aire, tierra, fuego, o la misma voz elemento), y la actualización y mejora de nuevos términos de la química (gas, oxígeno, ácido, metal, etc.) (Garriga 2018). Y también destacan las voces de la economía, con la entrada de capital capitalista, economista, empresario, circulación, balanza de comercio, etc. (De Hoyos, 2018). Por último, las voces de la navegación muestran, por un lado, el afán de recuperar los términos tradicionales, y por otro la necesidad de incorporar algunos neologismos surgidos de las innovaciones técnicas de fines del siglo XVIII y comienzos del XIX, aunque Carriazo (2018: 354) destaca de nuevo las supresiones y la labor de revisión llevada a cabo por la Academia. Y a todos estos datos, aún cabría añadir los del léxico de la electricidad, que incorpora seis nuevas acepciones a la edición: ámbar, electrizar ( $2^{\mathrm{a}}$ acepc.), electro, fluido electrico, fluido galvánico y pararayo (Moreno Villanueva 2012: 412). 
Las ediciones siguientes no han sido estudiadas tan pormenorizadamente, aunque cada vez se van conociendo mejor. Sabemos que en la $6^{\mathrm{a}}$ edición (RAE 1822) se produce la reducción ya comentada de más de dos mil entradas, a pesar del aumento de 517 lemas (Terrón, 2019: 742), lo que demuestra la importancia de las supresiones realizadas. Con todo, las voces especializadas vuelven a estar a la cabeza del aumento. Terrón (2019: 747) contabiliza 26 incorporaciones con marca diatécnica (de nuevo destacan la náutica, la medicina y la química) pero son muchas más las recién incorporadas que se pueden considerar especializadas aunque no lleven la marca correspondiente, así como las referidas a oficios y a nombres de plantas, animales y minerales. En la $7^{\mathrm{a}}$ (RAE 1832), como se ha apuntado, la reducción neta de voces es aún mayor, superando las seis mil entradas. A pesar de ello, entre las escasas incorporaciones destacan una vez más las voces especializadas, y particularmente las de náutica y química (Carriet 2017: 42), procedentes probablemente del diccionario de Núñez de Taboada (1825) (Clavería 2016: 106).

En las tres ediciones que cierran este período se recupera el incremento neto del número de voces, aunque de manera exigua. En la $8^{a}$ edición (RAE 1837) la nomenclatura académica crece, aunque apenas en algo más de quinientas palabras, de las que casi la mitad pertenecen al Suplemento ${ }^{4}$. Clavería (2016: 112) destaca ámbitos como la minería, la química y la música, aunque también señala la botánica, la medicina o el comercio, actividades a las que se vinculan las incorporaciones que se producen. La 9a edición (RAE 1843) registra un aumento de apenas 462 entradas, pocas de las cuales pertenecen a ciencias y artes (Clavería 2016: 123), lo que contradice las expectativas que podía despertar lo dicho en el prólogo. Y una situación parecida se produce en la $10^{\mathrm{a}}$ edición (1852), que registra un incremento neto de 545 entradas. No obstante, Clavería (2016: 132) destaca un mayor interés de la Academia por incorporar "palabras modernas", por lo que será de nuevo el léxico de la ciencia y de la técnica el mejor representado en el aumento del diccionario.

Estas percepciones se pueden ratificar en los estudios sobre determinados vocabularios que se han realizado en el seno del Grupo Neolcyt. Por ejemplo, sobre el léxico de la electricidad, los trabajos de Moreno Villanueva (2012a: 560) y (2012b: 482) muestran que en las ediciones posteriores a la $5^{\mathrm{a}}$ (RAE 1817) tan solo se registra la inclusión de galbanismo [sic] (RAE 1822), metal en su acepción eléctrica (RAE 1832), galbánico (RAE 1837), centella (RAE 1843), y galvanizar (2 acepciones), pararrayos y telégrafo eléctrico (RAE 1852). En el caso del léxico del ferrocarril, este no está representando en el diccionario académico hasta la $10^{\mathrm{a}}$ edición, donde entran camino de hierro, ferrocarril y locomotor (Rodríguez Ortiz, 2003). Y también en esta misma $10^{\mathrm{a}}$ edición aparecen las voces significativas del léxico de la fotografía: cámara oscura, daguerrotipia y daguerrotipo, fotografía, fotográfico

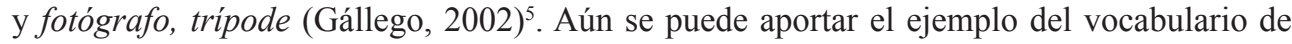
los alcaloides propios de la química orgánica, representado únicamente por la voz quinina (RAE 1837).

Se puede pensar que estas voces pertenecen a ciencias y técnicas que se desarrollan o incluso que irrumpen con fuerza en el siglo XIX, pero lo cierto es que eran palabras que ya

4 Según los datos obtenidos del NTLLE por Octavio Pinillos, la $8^{\text {a }}$ edición contiene 50.987 entradas en el cuerpo del diccionario, y 249 en el Suplemento.

5 Ciertamente desde la $4^{\mathrm{a}}$ edición (RAE 1803) se registran voces relacionadas con los productos que se utilizan en la fotografía, como algodón, emulsión, esencia, precipitado, solución, o con la elaboración: baño-maría, iluminación, nivelar, refinar, etc. (Gállego 2002). 
hacía tiempo que estaban en los textos, porque en los años inmediatamente anteriores a esta etapa la ciencia española había progresado considerablemente, y las traducciones de muchos textos científicos y técnicos habían naturalizado muchas de estas voces en español. Moreno Villanueva (1996) y (1997) estudia cómo el Ensayo sobre la electricidad de los cuerpos, del abad Nollet (1746) se traduce al español solo un año después de su publicación en francés, y en ella aparece ya una gran parte de estos términos. Rodríguez Ortiz (1998) analiza la lengua de los primeros proyectos y textos legislativos sobre el ferrocarril aparecidos en los comienzos del siglo XIX, así como las noticias de prensa de la época e incluso textos literarios de autores como Salas y Quiroga, Mesonero Romanos y Modesto Lafuente, y descubre que muchas las voces del ferrocarril son ya de uso frecuente. Igualmente, Gállego Paz (2002) y (2003) estudia los textos sobre fotografía que habían llegado tempranamente al español; entre ellos el Manual de Daguèrre, publicado en 1839 en francés, del que hay hasta tres versiones en español publicadas ese mismo año, las realizadas por Eugenio de Ochoa, Pedro Mata y Fontanet, y Joaquín Hysern y Molleras. Lo mismo se puede decir del vocabulario de la química orgánica, ya que el primer alcaloide se sintetiza en 1817, y ya en 1822 se documenta morfina en los Elementos de química aplicada a la medicina, la farmacia y las artes de Buenaventura Mateo Orfila (Garriga 2015), palabra que no entrará en el diccionario académico hasta la $11^{\mathrm{a}}$ ed. (RAE 1869), junto a nicotina, y a partir de ahí, el resto de los alcaloides (Garriga 2001).

Y este también es el caso del término incubación, utilizado en el título de este artículo. La palabra estaba en el diccionario desde el suplemento de la $8^{a}$ edición (RAE 1837) como 'el acto de empollar los huevos la gallina y otros ovíparos', pero adquiere un significado más especializado en el campo de la medicina que se documenta ya en 1822, en el Periódico de la Sociedad Médico-Quirúrgica de Cádiz (p. 105):

[...] puede generalizarse su uso cuando se echa de vér que la incubacion de la escarlatina, sarampión y de cualquiera otra flegmasía cutanea aguda está detenida por la languidez de las fuerzas vitales; lo mismo se verifica en los casos de metastasis, \&c.

Así lo recoge el Diccionario nacional de Domínguez (1846-47):

incubación [...] || Med. Período de incubacion: espacio de tiempo que transcurre desde que empieza á actuar el gérmen de una enfermedad, ó causa morbífica que obra sobre la economía animal, hasta que se preséntan los primeros síntomas.

Y solo aparece en el diccionario académico a partir de la $13^{\mathrm{a}}$ edición (RAE 1899):

Incubación. [...] || Med. Desarrollo de una enfermedad, desde que empieza á obrar la causa morbosa hasta que se manifiestan sus efectos.

Todos estos datos, aunque parciales, demuestran que la ciencia española, a pesar de la crisis política, económica y social de comienzos del siglo XIX, estaba latente, y que su vocabulario discurría por debajo del diccionario académico. El repertorio oficial, casi paralizado por la propia crisis de la Corporación, actuaba además como una especie de tapón que solo permitía aflorar una parte pequeña de ese vocabulario especializado, que aun así se convertía en el módulo léxico más dinámico del diccionario. Todo ello producía una sensación de 
abandono de la actividad científica y técnica, y de escasez de vocabulario, aún mayor de lo que ya era. Las voces técnicas se iban multiplicando en el español, sin encontrar un cauce que les permitiera canalizarse normativamente, hasta que esa presión desemboca primero en los diccionarios no académicos de Salvá, Domínguez, Chao, etc., y después en las ediciones académicas de la segunda mitad del siglo XIX. De nuevo la historia de la ciencia puede ayudar a interpretar qué pasa con la lengua de la ciencia y de la técnica en español.

\section{LA HISTORIA DE LA CIENCIA COMO INTERPRETACIÓN}

La ciencia moderna entra en las aulas universitarias en el siglo XIX. Peset (1988: 18) señala las tres líneas por las que se encaminaba la universidad ya en la Ilustración: la modernización, la uniformidad y la centralización, con la idea de controlar el poder universitario, hasta entonces en mano de los colegios, las órdenes, los claustros, etc. Se reforman los colegios mayores, se expulsa a los jesuitas y se introduce el libro de texto.

Las Cortes de Cádiz pretendían profundizar en ese proceso, rescatando la universidad de manos de la Iglesia y elevando el nivel científico. Esos objetivos se vieron obstaculizados por el retorno de Fernando VII, quien hizo retroceder de nuevo la universidad mediante los esquemas del Antiguo Régimen (Peset y Peset 1992: 22). El trienio liberal ideó un proyecto de inspiración francesa, en el que las ciencias se impartían en escuelas especiales, en particular las ciencias y las técnicas: medicina y cirugía, veterinaria, comercio, astronomía, navegación; a estas se sumaba una politécnica: artillería, ingenieros, minas, puentes y caminos, etc. Pero el proyecto quedó prácticamente en el papel, porque en 1824 el regreso del absolutismo barrió de nuevo los saberes científicos de la universidad. Las reformas se fueron sucediendo sin demasiado éxito a partir de 1833, con el reinado de Isabel II, hasta la Ley Moyano (1857).

La situación descrita en cuanto a la universidad y la enseñanza es paralela a la que se produce respecto al cultivo de las ciencias. A pesar del impulso recibido en la etapa de Carlos III, que sitúa la ciencia española en un buen nivel, una vez más el camino de la recuperación se ve truncado. López Piñero (1992: 14) lo expresa con las siguientes palabras:

En vísperas de la constitución de la ciencia contemporánea, España parecía a primera vista preparada para ser uno de sus focos iniciales. La realidad iba a ser, no obstante, muy distinta, ya que nuestra sociedad permanecería al margen de tan decisivo proceso, siendo después necesaria una penosa y prolongada aculturación para que fuera asimilando algunas de sus consecuencias.

Este autor identifica tres etapas en la ciencia a partir del siglo XIX: el "período de catástrofe" (1808-1833), la "etapa intermedia" (1834-1868), y la "generación de sabios" (18681936). Las dos primeras tocan de lleno el período aquí estudiado, y aunque se han trazado tomando la historia de la medicina como base, son representativas de toda la actividad científica, y pueden servir también para explicar qué pasa con la ciencia en el diccionario.

El "período de catástrofe" se explica en parte por la guerra, pero este hecho se dio en toda Europa, y contribuyó, con la reconstrucción, a crear nuevas oportunidades para la ciencia. Sin embargo en España no se produjo ese efecto. En ese momento era un país arruinado y sin prestigio internacional, situación ante lo cual se dieron dos posturas: la de los que consideraban un error mantener el esfuerzo de los ilustrados para renovar y europeizar las 
estructuras, y los partidarios de proseguir, desde posturas liberales. Como dice López Piñero (1992: 15), "[c]asi todas las instituciones científicas desaparecieron o vegetaron de modo lamentable". Con la excepción del "trienio liberal", se obstaculizó la comunicación con las novedades europeas y la circulación de publicaciones científicas. Esta situación afectó a los científicos, que vieron truncadas sus investigaciones, y que en muchos casos murieron o debieron exiliarse.

La "etapa intermedia" mejoró algo la situación anterior, aunque las condiciones para el cultivo de la ciencia siguieron siendo difíciles. La vuelta de los exiliados sirvió para que trajeran consigo las ideas científicas europeas y a partir de aquí iniciar una lenta mejoría.

En lo que respecta a las técnicas, sabemos que cada rama tiene desarrollos particulares, como se puede comprobar en los dos volúmenes dedicados al siglo XIX en la monumental Técnica e ingeniería en España coordinada por Silva Suárez (2011) y (2013), pero también se pone de manifiesto que es a partir de la segunda mitad del siglo cuando se empieza a remontar la situación de penuria y atraso, con el desarrollo tímido de la industria, la construcción de infraestructuras y obra pública, el desarrollo de las comunicaciones, etc.

Los datos sobre las publicaciones son un buen índice de la actividad científica de una época, y así se puede observar en el gráfico que resume el estudio bibliográfico realizado por Portela (1998: 50) sobre los textos de química publicados en España entre 1776 y 1900, donde se puede comprobar el bache que se produce en la primera mitad del siglo $\mathrm{XIX}^{6}$, y que solo empieza a remontar a partir de 1840.

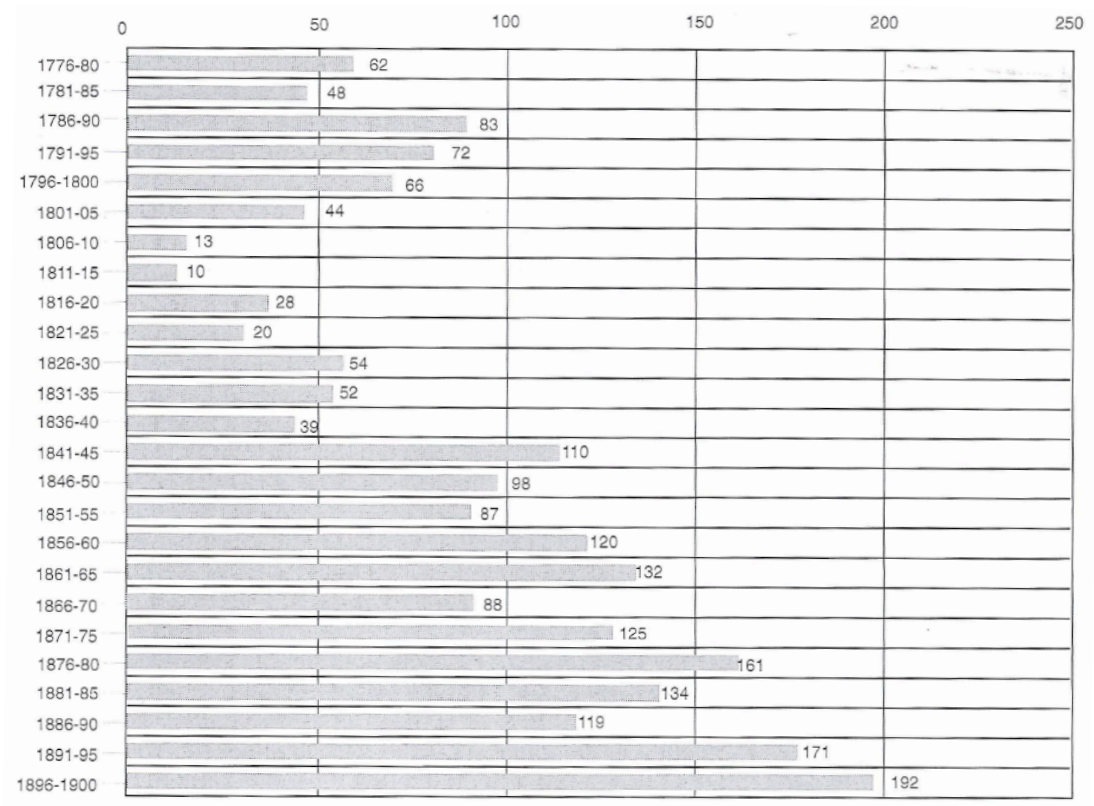

6 Portela (1998: 51) aclara que los datos del "período de catástrofe" aún serían más negativos si se descontaran las obras de Orfila, un químico mallorquín, de gran relevancia en los inicios de la toxicología en Francia, quien escribía desde París y en francés. 
Estas valoraciones y estos datos no sería difícil transponerlos a la actividad académica de esos años, especialmente entre la $6^{\mathrm{a}}$ edición (RAE 1822) y la $9^{\mathrm{a}}$ (RAE 1843), período en el que se podría afirmar, utilizando la citada idea de López Piñero (1992: 15), que la Academia como institución "vegetó de modo lamentable", tal como demuestran los datos que conocemos sobre las ediciones, y coincidiría también su recuperación con la propia de la ciencia española.

\section{CONCLUSIÓN}

Se puede considerar que la historia de las ediciones académicas no es diferente a la de otras actividades científicas y técnicas, y en especial la presencia de las voces especializadas es reflejo, por un lado, de la postura conservadora y restrictiva respecto a la inclusión de los tecnicismos en los diccionarios, y por otro de la parálisis de la Academia como institución que dificulta los normales trabajos de revisión de las ediciones de la primera mitad del siglo XIX.

Las dos primeras ediciones del siglo (RAE 1803 y RAE 1817) aún mantienen cierto tono de renovación, al abrigo del impulso que le proporcionan los trabajos para la segunda edición de Autoridades. La actividad académica se ralentiza al mínimo en las ediciones siguientes, y solo en la $10^{\mathrm{a}}$ (RAE 1852) parece remontar tímidamente.

A pesar de ello, la lengua de la ciencia, aunque lastrada por las dificultades que acuciaban la actividad científica, seguía su curso, como demuestran los textos y los ejemplos citados de algunas de las ciencias y técnicas estudiadas. Se va incubando todo un vocabulario que no tarda en encontrar salida en las ediciones académicas, de la misma manera que se va creando el caldo de cultivo político y social que acabará con el derrumbe de las viejas estructuras políticas y sociales en el siglo de las revoluciones.

En el caso de la ciencia, López Piñero señala la etapa posterior a 1868 como el momento en que se remonta decididamente la situación, igual que sabemos que es a partir de la $11^{\text {a }}$ edición (RAE 1869) cuando los neologismos de le ciencia y de la técnica empiezan a entrar en las páginas del diccionario académico, creando una especie de revolución lexicográfica -un "cambio de paradigma", en palabras de Clavería (2016: 170)- con la incorporación de cientos de tecnicismos que habían estado esperando en la antesala de los textos.

\section{REFERENCIAS BIBLIOGRÁFICAS}

Alvar Ezquerra, M. (1987). "Presentación". En Terreros, E. Diccionario castellano con las voces de ciencias y artes (ed. facsímil). Madrid: Arco Libros, pp. V-XVI.

Alvar Ezquerra, M. (2002). "El diccionario de la Academia en sus prólogos". De antiguos y nuevos diccionarios del español. Madrid: Arco Libros, pp. 253-286.

Álvarez de Miranda, P. (2001). "La lexicografía académica de los siglos XVIII y XIX". En Ahumada, I. (coord.). pp. 35-62.

Álvarez de Miranda, P. (2011). "Vicente Salvá y la lexicografía española de la primera mitad del siglo XIX”. Los diccionarios del español moderno. Gijón: Ediciones Trea, pp. 89-118.

Azorín, D. (2003a). "Un proyecto original en la lexicografía española del siglo XIX: el Nuevo Diccionario de la Lengua Castellana (1846) de Vicente Salvá”. En Echenique, M. T. y J. Sánchez Méndez (eds.). Lexicología y Lexicografía en Europa y América. Homenaje a Günther Haensch. Gredos: Madrid, pp. 115-131.

Azorín, D. (2003b). "Neologismos incorporados por Vicente Salvá en su Nuevo Diccionario de la Lengua Castellana". Estudios de Lingüistica de la Universidad de Alicante, 17, pp. 107-139. 
Azorín, D. y Ma I. Santamaría (2004). "El Diccionario de autoridades (1726-1739) y el Diccionario castellano (1786-1793) de Terreros y Pando ante la recepción de las voces de especialidad". Revista de Investigación Lingüística, 7, pp. 49-70.

Bails, B. (1802). Diccionario de arquitectura civil. Madrid: Viuda de Ibarra.

Brisson, M. T. (1796-1802). Diccionario Universal de Física (10 vols.) (trad. C. Cladera y F. X. C.). Madrid: Benito Cano-Imprenta Real.

Capmany, A. de (1817). Nuevo diccionario francés español. Madrid: Imprenta de Ibarra.

Carriazo, J. R. (2018). "Las voces de la navegación: náutica, ingeniería naval y áreas afines". En Clavería, G. y M. Freixas (coords.), pp. 339-355.

Carriet, E. (2017). "La séptima edición del diccionario académico (DRAE-1832)". Revista de lexicografia, XXIII, pp. 39-65.

Clavería, G. (2016). De vacunar a dictaminar: la lexicografía académica decimonónica y el neologismo. Madrid - Frankfurt am Main: Iberoamericana - Vervuert.

Clavería, G. (2018). "La quinta edición del Diccionario de la lengua castellana (1817) de la Real Academia Española al microscopio". En Clavería, G. y M. Freixas (coords.), pp. 15-55.

Clavería, G. y M. Freixas (coords.) (2018). El diccionario de la Academia en el siglo XIX; la $5^{a}$ edición (1817) al microscopio. Madrid: Arco Libros.

De Hoyos, J. C. (2018). "Las voces de la economía política”. En Clavería, G. y M. Freixas (coords.), pp. 357-370.

DICTER. Diccionario de la ciencia y de la técnica del Renacimiento. $\mathrm{M}^{\mathrm{a}}$ Jesús Mancho Duque (dir.). Ediciones Universidad de Salamanca. http://dicter.usal.es/ (12-12-2018).

Domínguez, J. R. (1846-47). Diccionario nacional o gran diccionario clásico de la lengua española. Madrid: Establecimiento tipográfico de R. J. Domínguez

Esparza, M. Á. (1999). "Notas sobre el Diccionario Nacional de Ramón Joaquín Domínguez". En Henríquez, M., C. y M. Á. Esparza (eds.). Estudios de historiografía lingüística hispánica ofrecidos a Hans-Josef Niederehe. Vigo: Universidad de Vigo, pp. 39-63.

Freixas. M. (2010). Planta y método del Diccionario de autoridades. Orígenes del método lexicográfico de la Real Academia Española (1713-1739). A Coruña: Universidade da Coruña.

Galera, A. (2002). "La corte ilustrada de Carlos III". En Peset, J. L. (dir.). Historia de la ciencia y de la técnica en la Corona de Castilla (siglo XVIII). Salamanca: Junta de Castilla y León, pp. 49-64.

Gállego, R. (2002). "Notas sobre la historia del léxico de la fotografía en español”. En Echenique, $\mathrm{M}^{\mathrm{a}}$ T. (eds.). Actas del V Congreso Internacional de Historia de la Lengua Española. Madrid: Gredos, pp. 2051-2062.

Gállego, R. (2003). "El léxico de la fotografía en los textos del siglo XIX en España”. Asclepio, LV-2, pp. 135-157.

García-Macho, Ma L. (2014). "Los diccionarios especializados o técnicos del Siglo de Oro". Anuario de Estudios Filológicos, XXXVII, pp. 71-89.

Garriga, C. (1998). "El Diccionario Universal de Física de Brisson (1796-1802) y la fijación lexicográfica de la terminología química en español". En García Turza, C. et al. (eds.). Actas del IV Congreso Internacional de Historia de la Lengua Española. Logroño: AHLE - Gobierno de la Rioja - Universidad de La Rioja, pp. 179-190.

Garriga, C. (2001). "Notas sobre el vocabulario de la química orgánica en español: Liebig y la divulgación de los derivados en -ina". En Bargalló, M., E. Forgas, C. Garriga, J. Schnitzer y A. Rubio (eds.). Las lenguas de especialidad y su didáctica. Tarragona: Universitat Rovira i Virgili, pp. $169-180$.

Garriga, C. (2002). "Notas sobre la incorporación de los términos de elementos químicos al español en el siglo XIX: el Léxico histórico y sinonímico de F. Hoefer". En Pöll, B. y F. Rainer (eds.). Vocabula et vocabularia: Études de lexicologie et de (méta-)lexicographie romanes en lihonneur du 60è anniversaire de Dieter Messner. Frankfurt am Main: Peter Lang, pp. 131-144. 
Garriga, C. (2003). "La química y la lengua española en el siglo XIX”. Asclepio, LV / 2, pp. 93-117. Garriga, C. (2015). "Aspectos de la traducción científica en el siglo XIX: el ejemplo de Orfila". Traducción y difusión de la ciencia y la técnica en España (siglos XVI-XIX). Valencia: Universitat de València, pp. 229-248.

Garriga, C. (2018). "Las voces de la química”. En Clavería, G. y M. Freixas (coords.), pp. 313-337.

Garriga, C. y S. Iglesia (2010). "La técnica lexicográfica española del siglo XIX: el Diccionario Nacional de R. J. Domínguez y la lengua de la ciencia y la técnica", Beiträge zur Geschichte der Sprachwissenschaft, 20.2, pp. 227-238.

Gómez de Enterría, J. (2018). "Las voces de las ciencias naturales y áreas afines”. En Clavería, G. y M. Freixas (coords.), pp. 275-311.

Iglesia, S. (2008). "El Diccionario de R. J. Domínguez como ejemplo de la influencia de la lexicografía francesa en la lexicografía española del siglo XIX". En Azorín, D. et al. (eds.). El diccionario como puente entre las lenguas y culturas del mundo. Actas del II Congreso Internacional de Lexicografía Hispánica. Alicante: Universidad de Alicante, pp. 121-127.

Iglesia, S. (2011). "El Diccionario (1846-1847) de Ramón Joaquín Domínguez". En San Vicente, F., C. Garriga y H. E. Lombardini (coords.). IDEOLEX. Estudios de lexicografía e ideología. Polimetrica: Monza, pp. 419-438.

Iglesia, S. (2012). "La obra lexicográfica de Domínguez: del Diccionario francés-español y del Diccionario Nacional”. En Nomdedeu, A., E. Forgas y M. Bargalló (eds.), pp. 343-355.

Jiménez Ríos, E. (2018). "El léxico con marcación diacrónica”. En Clavería, G. y M. Freixas (coords.), pp. 427-453.

Lázaro Carreter, F. (1949 [1985]). Las ideas lingüisticas en España durante el siglo XVIII. Barcelona: Crítica.

López Piñero, J. M. (1992). "Introducción”. Ayer, 7, (nº monográfico "La ciencia en la España del siglo XIX”, ed.: J. Ma López Piñero), pp. 11-19.

López Piñero, J. M. (dir.) (2002). Historia de la ciencia y de la técnica en la Corona de Castilla (siglos XVI y XVII). Salamanca: Junta de Castilla y León.

Mancho, M. J. (2008). "La divulgación técnica: características lingüísticas". En Silva Suárez, M. (ed.). Técnica e ingeniería en España I: El Renacimiento. De la técnica imperial y la popular. Zaragoza: Real Academia de Ingeniería - Institución Fernando el Católico - Prensas Universitarias de Zaragoza, pp. 311-344.

Moreno Villanueva, J. A. (1996). "Jean-Antoine Nollet y la difusión del estudio de la electricidad: un nuevo léxico para una nueva ciencia”, Documents pour l'histoire du français langue étrangère ou seconde, 18, pp. 405-417

Moreno Villanueva, J. A. (1997). "El Essai sur l'électricité des corps (1746) de J.-A. Nollet: primer texto sobre física eléctrica traducido al español”. Grenzgänge, 4, pp. 17-32.

Moreno Villanueva, J. A. (2012a). "La inclusión del léxico de la electricidad en los diccionarios de mediados del siglo XIX". En Nomdedeu, A., E. Forgas y M. Bargalló (eds.), pp. 411-425.

Moreno Villanueva, J. A. (2012b). "Notas sobre el vocabulario de la electrostática en el siglo XVIII: los primeros generadores de electricidad”. En Rio-Torto, G. (ed.). Léxico de la ciencia: tradición $y$ modernidad. Muenchen: Lincom Europa, pp. 332-348.

Morveau, L. G., A. L. Lavoisier, Cl. Berthollet y A. F. Fourcroy (1788). Método de la nueva nomenclatura química (trad. de P. Gutiérrez Bueno). Madrid: Imprenta de Sancha.

Morveau, L. G., H. Maret y J. F. Durande (1788). Elementos de química teórica y práctica (trad. de M. de Guardia y Ardévol). Madrid: Benito Cano.

NTLLE: RAE (en línea). Nuevo Tesoro Lexicográfico de la Lengua Española. http://ntlle.rae.es (1112-2018).

Nomdedeu, A., E. Forgas y M. Bargalló (eds.). Avances de lexicografía hispánica, I. Tarragona: Publicacions URV. 
Pascual Fernández, M. L. (2013). Diccionario de autoridades (2. ${ }^{a}$ ed.): técnica lexicográfica y lengua de la ciencia, tesis doctoral. Bellaterra: Universitat Autònoma de Barcelona, disponible en $<\mathrm{http}: / /$ ddd.uab.cat/record/115331>.

Peset, J. L. (1988). "Educación y ciencia en el fin del Antiguo Régimen”. En Sánchez Ron, J. M. (ed.). Ciencia y sociedad en España: de la Ilustración a la Guerra Civil. Madrid: El Arquero / CSIC, pp. 17-25.

Peset, J. L. (dir.) (2002). Historia de la ciencia y de la técnica en la Corona de Castilla (siglo XVIII). Salamanca: Junta de Castilla y León.

Peset, M. y J. L. Peset (1992). "Las universidades españolas del siglo XIX y las ciencias". Ayer, 7, ( $n^{\circ}$ monográfico "La ciencia en la España del siglo XIX", ed.: J. Mª López Piñero), pp. 19-51.

Portela, E. (1998). La química en el siglo XIX. Madrid: Akal.

Quilis Merín, M. (2007). "La tareas lexicográficas del siglo XIX: los diccionarios de Ramón Joaquín Domínguez”. En Campos, M., R. Cotelo García y J. I. Pérez Pascual (eds.). Historia de la lexicografía española. La Coruña: Universidade da Coruña, pp. 141-152.

Raab, M. (2018). "La lexicografía no académica y la quinta edición del DRAE". En Clavería, G. y M. Freixas (coords.), pp. 529-542.

RAE (1726-1739). Diccionario de la lengua castellana, en que se explica el verdadero sentido de las voces, su naturaleza y calidad con las phrases o modos de hablar, los proverbios o refranes, y otras cosas convenientes al uso de la lengua. Madrid: Imprenta Francisco del Hierro.

RAE (1743). Reglas, que formó la Academia en el año de 1743 y mandó observassen los señores Académicos, para trabajar con uniformidad en la correccion, y Suplemento del Diccionario, [s. 1., sin pie de imprenta].

RAE (1757). Nuevas reglas que ha formado la Academia Española para la correccion y aumento del Diccionario. Año 1757. Manuscrito 415 de la Biblioteca de la Real Academia Española.

RAE (1791). Diccionario de la lengua castellana, reducido a un solo tomo para su más fácil uso, tercera edición. Madrid: Viuda de don Joaquín Ibarra.

RAE (1803). Diccionario de la lengua castellana, reducido a un solo tomo para su más fácil uso, cuarta edición. Madrid: Viuda de don Joaquín Ibarra.

RAE (1817). Diccionario de la lengua castellana, quinta edición. Madrid: Imprenta Real.

RAE (1822). Diccionario de la lengua castellana, sexta edición. Madrid; Imprenta Nacional.

RAE (1832). Diccionario de la lengua castellana, séptima edición. Madrid: Imprenta Real.

RAE (1837). Diccionario de la lengua castellana, octava edición. Madrid: Imprenta Nacional.

RAE (1843). Diccionario de la lengua castellana, novena edición. Madrid: Imprenta de D. Francisco María Fernández.

RAE (1852). Diccionario de la lengua castellana, décima edición. Madrid: Imprenta Nacional.

RAE (1869). Diccionario de la lengua castellana, undécima edición. Madrid: Imprenta de Don Manuel Rivadeneyra.

RAE (1899). Diccionario de la lengua castellana, décimatercia edición. Madrid: Imprenta de los Sres. Hernando y compañía.

Rodríguez Ortiz, F. (1998). "Tratados ferroviarios en España: primeras traducciones”. En García, J. L., J. M. Moreno y G. Ruiz (eds.). Estudios de historia de las técnicas, la arqueología industrial y las ciencias. Segovia: Junta de Castilla y León, pp. 449-455.

Rodríguez Ortiz, F. (2003). "La lengua y la técnica en el siglo XIX: el ejemplo del ferrocarril". Asclepio, LV / 2, pp. 119-133.

Rodríguez Ortiz, F, y C. Garriga (2010). "La teoría lexicográfica de la Academia en los siglos XVIII y XIX a través de las reglas". Quaderns de filología. Estudis lingüistics, 15, pp. 31-56.

Salvá, V. (1846). Nuevo diccionario de la lengua castellana. París: Librería D. Vicente Salvá.

San Vicente, F. y H. Lombardini (2012). "Prólogos del DRAE (1780-2001): cánones formales y de contenido”. En Nomdedeu, A., E. Forgas y M. Bargalló (eds.), pp. 479-493. 
Seco, M. (1987). "El nacimiento de la lexicografía moderna no académica”. Estudios de lexicografía española. Madrid: Paraninfo, pp. 129-151.

Silva Suárez, M. (ed.) (2011). Técnica e ingeniería en España - VII: El Ochocientos - De los lenguajes al patrimonio. Zaragoza: Real Academia de Ingeniería - Institución Fernando el Católico - Prensas universitarias de la Universidad de Zaragoza.

Silva Suárez, M. (ed.) (2013). Técnica e ingeniería en España - VII: El Ochocientos - De las profundidades a las alturas. Zaragoza: Real Academia de Ingeniería - Institución Fernando el Católico - Prensas universitarias de la Universidad de Zaragoza.

Terreros, E. (1786). Diccionario castellano con las voces de ciencias y artes, ed. facsímil. Madrid: Arco Libros, 1987.

Terrón, N. (2019). "Historia de la lengua y lexicografía: el aumento de voces en la 6. ${ }^{a}$ edición del Diccionario de la Academia (1822)". En Arnal, M. ${ }^{a}$ L. et alii (eds.). Actas del X Congreso Internacional de Historia de la Lengua Española. Zaragoza: Institución Fernando el Católico, vol. II, pp. 1325-1341. 